\title{
Correction to: Efficacy of a medication management service in improving adherence to tyrosine kinase inhibitors and clinical outcomes of patients with chronic myeloid leukaemia: a randomised controlled trial
}

\author{
Bee Kim Tan ${ }^{1}$ (D) Siew Siang Chua ${ }^{2} \cdot$ Li-Chia Chen $^{3} \cdot$ Kian Meng Chang ${ }^{4,5} \cdot$ Sharmini Balashanker $^{6} \cdot$ Ping Chong Bee $^{1}$
}

Published online: 7 December 2019

(C) Springer-Verlag GmbH Germany, part of Springer Nature 2019

Correction to: Supportive Care in Cancer

https://doi.org/10.1007/s00520-019-05133-0

The correct Funding Information should be:

This study received funding from research grants from the University of Malaya (PG057-2015A) and the Malaysian Society of Haematology (MSHRFC-2017-001).

The online version of the original article can be found at https://doi.org/ 10.1007/s00520-019-05133-0

Ping Chong Bee

pcbee@um.edu.my

1 Department of Medicine, Faculty of Medicine, University of Malaya, Jalan Universiti, 50603 Kuala Lumpur, Malaysia

2 School of Pharmacy, Faculty of Health and Medical Sciences, Taylor's University, 47500 Subang Jaya, Selangor, Malaysia

3 Centre for Pharmacoepidemiology and Drug Safety, Division of Pharmacy and Optometry, School of Health Sciences, Faculty of Biology, Medicine and Health, University of Manchester, Manchester Academic Health Science Centre, Manchester M13 9PT, UK

4 Department of Hematology, Ampang Hospital, 68000 Ampang, Selangor, Malaysia

5 Ministry of Health Malaysia, Putrajaya, Malaysia

6 School of Pharmacy, University of Nottingham Malaysia Campus, 43500 Semenyih, Selangor, Malaysia 